\title{
Expectativas y creencias del alumnado rural sobre su futuro profesional y académico
}

\author{
Juan Lorenzo Lacruz*, Virginia Domingo Cebrián*, María Tomé Fernández ${ }^{* *}$ \\ * Departamento de Ciencias de la Educación. Universidad de Zaragoza \\ ** Departamento de Métodos de Investigación y Diagnóstico en Educación. Universidad de Granada
}

\section{RESUMEN}

Actualmente las escuelas rurales están siendo modelos alternativos de enseñanza en muchos puntos de España. Estas escuelas presentan ventajas educativas que no tienen las escuelas urbanas. ¿Pero qué opinan los alumnos de estas escuelas sobre su futuro académico y profesional? A pesar de que las investigaciones en este tema son escasas, en este estudio se pretenden conocer las expectativas y creencias del alumnado rural en cuanto a su futuro profesional y académico. Para ello, a través de un muestreo no probabilístico se seleccionó a 270 estudiantes correspondientes a escuelas rurales de Aragón, Cataluña y Andalucía. Para evaluar el objetivo planteado se elaboró un cuestionario ad hoc. Los resultados obtenidos se analizaron mediante el software estadístico SPSS en su versión 17. Entre estos resultados se encuentra que el 57, 8\% de los estudiantes evaluados considera que tendrán éxito en su futuro académico y a más del $50 \%$ le gustaría trabajar en un empleo perteneciente al sector servicios. Por último, entre las conclusiones más relevantes se halla que el número de habitantes de los pueblos en donde los alumnos viven influye en la creencia que los estudiantes tienen de ellos mismos y en el trabajo que creen que desempeñarán.

Palabras Clave: Escuela rural, creencias, expectativas, estudiantes rurales, pueblos españoles.

\section{Expectations and beliefs on rural student about their professional and academic future}

\section{ABSTRACT}

Nowadays, many Spanish rural schools have become alternative teaching models. These schools have several advantages over urban schools. Our interest lies in the opinions of the rural students on their academic and professional future. Thus, we intend to know their expectations and beliefs through a probabilistic sampling over 270 students of rural schools of several Spanish regions (Aragon, Cataluña and Andalucia). For that purpose we designed a questionnaire and the results were analyzed with SPSS statistic software. Among the results we came across that $57,8 \%$ of the students think they will be successful in their academic future and more than $50 \%$ would like to work in a profession related to tertiary sector. Finally, we found that there is a straight relationship between the population of the villages and the beliefs and expectations the students have of themselves.

Keywords: Rural school, students' beliefs, expectations, students, Spanish villages.

\section{Introducción}

Las diferentes coyunturas políticas y sociales de finales del siglo XX y el esfuerzo de diferentes profesionales de la educación ( $\underline{\mathrm{Feu}, 2004}$ ) sentaron las bases de la escuela rural como la conocemos en la actualidad. La inmigración, la sociedad de la información, así como la nueva estructura de los centros y la situación del profesorado actual (Murillo, 2007), han modificado la concepción de escuela rural tradicional y esta se ha convertido en un concepto mucho más complejo y polisémico (Bernal, 2009). Es una escuela capaz de afrontar las nuevas realidades educativas, influidas por la sociedad española actual, que se reflejan en la escuelas rurales con nuevas necesidades educativas (Manzano y Tomé, 2016).

En los últimos años, las condiciones de la escuela rural han ido mejorando progresivamente hasta convertirse en muchos puntos 
de España en una alternativa a la escuela urbana (Abós, 2007). La escuela rural actual se muestra como un modelo alternativo para frenar aspectos negativos de las escuelas urbanas (Sepúlveda y Gallardo, 2011) ya que se caracterizan por su diversidad en el alumnado, tanto en su edad como en su formación cultural, así como por el bajo ratio de estos, la eficaz atribución de recursos e infraestructuras a las necesidades educativas surgidas, la relación más directa entre familia y escuela, y por modelos organizativos concretos que hacen estas escuelas autónomas e independientes (Bernal, 2009).

A pesar de estas características, aparentemente ventajosas, la distribución y dispersión geográfica de las escuelas rurales hacen que muchas veces lo establecido por la Administración y la legislación educativa no se adapten al medio concreto en el que se sitúa un determinado centro. En esas situaciones el trabajo coordinado y contextualizado en un medio a priori complicado es fundamental para salvar las dificultades y aprovechar las oportunidades que ofrece. Se tratará de lograr "acomodarse a las necesidades e intereses de esta sociedad en constante movimiento, de esta sociedad líquida" (Boix, 2011, p.15).

Por todo ello, podemos decir que el medio rural condiciona sobremanera el modo en que se presta el servicio de la educación, pues de estos factores e influencias se derivan paradójicamente grandes dificultades y desafíos propios de las dificultades naturales, sociales, económicas y poblacionales, pero que sin embargo abren el camino de la experimentación flexible y autónoma. En definitiva, el medio rural ofrece grandes posibilidades que pueden ser explotadas a partir del compromiso educativo y sociopolítico, del esfuerzo conjunto y coordinado de las comunidades rurales y la implicación por hacer de la escuela rural un instrumento que garantice el mantenimiento de la sociedad rural, así como su transformación hacía nuevos horizontes.

En cuanto a las investigaciones relacionadas con las escuelas rurales, existen diversos estudios (Coladarci, 2007; Champollion, 2013) que plantean la dificultad de la investigación educativa relacionadas con la escuela rural. Bustos (2011) señala que al comparar la producción literaria actual sobre escuela rural con otros temas educativos como TIC, competencias básicas, convivencia, etc., se pone de manifiesto la desproporción existente sobretodo en el ámbito científico español.

De esta manera, las pocas investigaciones realizadas en este ámbito de estudio están relacionadas principalmente con la organización de la escuela rural y su presencia en la legislación educativa (Calvo, 2003; Gómez, 2007; Murillo, 2007 y Mallada, 2012) así como en una serie análisis históricos de la demografía en distintos medios rurales españoles y aragoneses (Sanz, 2009; Ayuda, Pinilla y Sáez, 2010; Benito, 2013) e internacionales (Williamson, 2010; Jean, 2007; Alpe y Fauguet, 2008; Kalaoja y Pietarinen, 2009; Alpe, 2012). Otras investigaciones configuran estudios de tipo histórico, sociológico y hermenéutico (Berlanga, 2003 y 2009; Bernat, 2004; Bernal, 2006 y 2009) y otras se centras en diversos análisis comparativos entre lo rural y lo urbano (Hopkins, 2005; Corbett, 2007). Finalmente, contamos con estudios que tratan de enmarcar y clarificar la pedagogía específica que puede potencialmente ponerse en marcha en contextos educativos rurales (Feu, 2004; González, 2010).

En esta investigación se parte de un marco teórico limitado, ya que es casi nulo el número de estudios en donde las creencias y expectativas del alumnado de las escuelas rurales hayan sido el objetivo principal. Tan solo un estudio realizado por Domingo (2013) trata este objetivo. En esta última investigación, antiguos alumnos de escuelas rurales turolenses responden a una serie de ítems en donde se destacan como conclusiones principales que la mayoría de este alumnado consideró la enseñanza individualizada de las escuelas rurales muy eficaz para su preparación académica en estudios posteriores. Así, los alumnos destacaban el hecho de que los maestros prestaban una atención más individualizada, que su paso por la escuela rural servía para aprender a convivir con alumnos de distintas edades y ritmos de aprendizaje y que, en bastantes casos, notaban que tenían más nivel que otros alumnos cuando se incorporaban a la siguiente etapa educativa.

Según los resultados obtenidos en la presente investigación con respecto a las expectativas y creencias sobre el futuro académico y profesional del alumnado de la escuela rural, se parte de las siguientes hipótesis:

H1: Los estudiantes de las escuelas rurales españolas creen que tienen un buen nivel escolar.

H2: Los alumnos de escuelas rurales creen que tendrán éxitos en sus estudios.

H3: Los estudiantes de escuelas rurales creen que podrán trabajar en trabajos relacionados con el sector servicios.

H4: El alumnado de escuelas rurales preferirá trabajar en profesiones que se desarrollen en ciudades grandes.

Estas hipótesis pretenden dar respuesta al objetivo principal del estudio: conocer las expectativas y creencias del alumnado rural en cuanto su futuro académico y profesional.

\section{Método}

\section{Participantes}

A la población de este estudio pertenecen 324 estudiantes de escuelas rurales correspondientes a las comunidades autónomas de Aragón (176), Cataluña (113) y Andalucía (35) durante el curso académico 2014-2015. Finalmente, tras realizarse un muestreo no probabilístico de tipo intencional, se trabajó en esta investigación con una muestra total de $(\mathrm{N}=270)$, componiendo la muestra andaluza 32 estudiantes, 88 la muestra catalana y 150 estudiantes la muestra aragonesa, tal y como se observa en la Tabla 1.

Tabla 1. Distribución de la muestra $(\mathrm{N}=270)$

\begin{tabular}{lc}
\hline Comunidad Autónoma & Número de alumnos \\
\hline Andalucía & 32 \\
Aragón & 150 \\
Cataluña & 88 \\
Total & 270 \\
\hline
\end{tabular}

Fuente: Elaboración propia.

En cuanto a las principales características socio-demográficas, el grupo muestral manifiesta una edad media de 11,40 años (44,90\% hombres y 55,10\% mujeres). El $80 \%$ de la muestra vive en localidades menores de 1000 habitantes y mayores de 200. El porcentaje de alumnado que asiste a centros escolares no agrupados es de $17,7 \%$ frente al $82,30 \%$ de los estudiantes que asisten a escuelas rurales agrupadas. El 45,34\% proviene de una familia donde la madre trabaja en el sector servicios y el padre en el sector primario. 


\section{Instrumento}

Tras una exhaustiva revisión teórica, se observó que no existía un instrumento que evaluase las creencias y expectativas académicas y profesionales de los estudiantes de las escuelas rurales. Por ello, se realizó un cuestionario ad hoc que plasmase las variables que se pretendía medir.

La elaboración del instrumento se llevó a cabo utilizando la técnica de juicios de expertos. Para ello se presentó el instrumento a 8 expertos en Educación Rural, pidiéndoles que valorasen del 1 al 5 en una escala tipo Likert la idoneidad de los ítems para analizar las expectativas académicas y profesionales del alumnado de escuelas rurales. Tras la valoración de los expertos, de los 36 ítems presentados, el cuestionario quedó compuesto finalmente por 12 ítems, que fueron las preguntas del cuestionario que obtuvieron una media de 3 o superior por los expertos. Los ítems que componen el instrumento son de respuestas tipo Likert del 1 al 3. El cuestionario resultante obtuvo un análisis de fiabilidad de $\alpha=0,85$.

\section{Procedimiento}

Se trata de una investigación ex post facto, explicativa y correlacional. El estudio se ha desarrollado desde un enfoque holístico, dada las características de las variables a evaluar.

La recogida de información se ha llevado a cabo mediante 2 procedimientos:

- Solicitud de permiso a los tutores legales de la muestra a evaluar.

- Aplicación del cuestionario directamente a la muestra o bien mediante la colaboración de los maestros.

Los análisis descriptivos y correlacionales se realizaron mediante el paquete estadístico SPSS en su versión 17.

\section{Resultados}

En la Tabla 2 se muestra el análisis descriptivo elaborado en esta investigación:

Tabla 2. Porcentajes de respuestas para cada uno de los ítems evaluados

\begin{tabular}{|c|c|c|c|}
\hline Ítems & & $\%$ Respuestas & \\
\hline Me gusta la escuela & $\begin{array}{l}\text { Mucho } \\
51,6\end{array}$ & $\begin{array}{c}\text { Poco } \\
41,3\end{array}$ & $\begin{array}{l}\text { Nada } \\
7,1\end{array}$ \\
\hline Cuál creo que es mi nivel escolar & $\begin{array}{l}\text { Bueno } \\
57,4\end{array}$ & $\begin{array}{l}\text { Mediano } \\
39,8\end{array}$ & $\begin{array}{l}\text { Con dificultades } \\
2,7\end{array}$ \\
\hline Cómo crees que conseguirás aprobar este curso & $\begin{array}{l}\text { Sin dificultad } \\
47,8\end{array}$ & $\begin{array}{l}\text { Lo conseguiré con dificultades } \\
99,5\end{array}$ & $\begin{array}{c}\text { No lo sé } \\
12,6\end{array}$ \\
\hline Creo que tendré éxitos en mis estudios & $\begin{array}{c}\text { Sí } \\
42,2\end{array}$ & $\begin{array}{l}\text { Quizás } \\
57,8\end{array}$ & $\begin{array}{c}\text { No } \\
0\end{array}$ \\
\hline Primera profesión en la que me gustaría trabajar & $\begin{array}{l}\text { Sector primario } \\
1,9\end{array}$ & $\begin{array}{l}\text { Sector secundario } \\
15,1\end{array}$ & $\begin{array}{l}\text { Sector terciario } \\
54,3\end{array}$ \\
\hline Segunda profesión en la que me gustaría trabajar & $\begin{array}{l}\text { Sector primario } \\
1,9\end{array}$ & $\begin{array}{l}\text { Sector secundario } \\
\qquad 15,8\end{array}$ & $\begin{array}{l}\text { Sector terciario } \\
51,5\end{array}$ \\
\hline Tercera profesión en la que me gustaría trabajar & $\begin{array}{l}\text { Sector primario } \\
1,6\end{array}$ & $\begin{array}{l}\text { Sector secundario } \\
16,9\end{array}$ & $\begin{array}{l}\text { Sector terciario } \\
\quad 54\end{array}$ \\
\hline Primera profesión en la que realmente creo que podré trabajar & $\begin{array}{l}\text { Sector primario } \\
\qquad 3,1\end{array}$ & $\begin{array}{l}\text { Sector secundario } \\
18,7\end{array}$ & $\begin{array}{l}\text { Sector terciario } \\
54,9\end{array}$ \\
\hline Segunda profesión en la que creo que podré trabajar & $\begin{array}{l}\text { Sector primario } \\
\qquad, 4\end{array}$ & $\begin{array}{l}\text { Sector secundario } \\
16,9\end{array}$ & $\begin{array}{l}\text { Sector terciario } \\
50,8\end{array}$ \\
\hline Tercera profesión en la que creo que realmente podré trabajar & $\begin{array}{l}\text { Sector primario } \\
\quad 30\end{array}$ & $\begin{array}{l}\text { Sector secundario } \\
18,8\end{array}$ & $\begin{array}{l}\text { Sector terciario } \\
55,6\end{array}$ \\
\hline Entorno en el que me gustaría trabajar en un futuro & $\begin{array}{l}\text { En el campo } \\
36\end{array}$ & $\begin{array}{c}\text { Ciudad pequeña } \\
29,10\end{array}$ & $\begin{array}{l}\text { Ciudad mediana o } \\
\text { grande } 41,80\end{array}$ \\
\hline Hasta qué edad debería seguir estudiando & $\begin{array}{l}\text { Hasta los 14-18 años } \\
\qquad 22,2\end{array}$ & $\begin{array}{c}\text { Hasta los } 18-22 \text { años } \\
40,6\end{array}$ & $\begin{array}{c}\text { Más de } 22 \\
37,2\end{array}$ \\
\hline
\end{tabular}

Fuente: Elaboración propia.

Tal y como se observa en la tabla anterior, al 51,60\% del alumnado de la escuela rural le gusta mucho la escuela, mientras que el 47,8 \% considera que conseguirá aprobar el curso sin problemas y el $57,8 \%$ cree que quizás tenga éxito en su futuro académico.

Con respecto a la profesión que querrán realizar en un futuro, a más del $50 \%$ les gustaría optar como primera, segunda y tercera opción a una profesión que perteneciese al sector servicios, creyendo que seguramente tendrán la opción real de conseguir desempeñar estas profesiones.

Por lo que respecta al entorno en el que le gustaría trabajar en un futuro, al $41,80 \%$ les gustaría desempeñar una profesión en una ciudad mediana o grande, frente al $36 \%$ que preferiría en el campo. Por otro lado, el 40,6\% cree que deberá seguir estudiando hasta los 18-22 años, un porcentaje ligeramente superior al de aquellos que creen que deberán continuar estudiando hasta más allá de los 22 años (37,2\%).

Para profundizar sobre estos datos se han realizado varios análisis descriptivos de tablas cruzadas.

Como se observa en la Tabla 3, el 79,2\% de los alumnos que viven en pueblos con más de 2000 habitantes se consideran buenos estudiantes, frente al $20 \%$ de los alumnos que se consideran buenos en aquellos pueblos menores de 200 habitantes.

Es en estos pueblos pequeños en donde también se observa más cantidad de alumnos con dificultades (20\%), con un porcentaje significativamente superior al obtenido en el resto de las poblaciones (200-500 1,5\%; 500-1000 2,3\%; 1000-2000 7,1\%; más de 2000 0\%). 
Tabla 3. Tabla cruzada entre tamaño de la población, consideración como alumno y éxitos académicos.

\begin{tabular}{|c|c|c|c|c|c|c|}
\hline \multirow[b]{3}{*}{ Tamaño de la población } & \multicolumn{6}{|c|}{$\%$ Respuestas } \\
\hline & \multicolumn{3}{|c|}{ Consideración como alumno } & \multicolumn{3}{|c|}{ Éxitos académicos } \\
\hline & $\begin{array}{l}\text { Buen } \\
\text { alumno }\end{array}$ & $\begin{array}{r}\text { Alumnos } \\
\text { mediano }\end{array}$ & $\begin{array}{l}\text { Alumno con } \\
\text { dificultades }\end{array}$ & $\begin{array}{c}\text { Lo conseguiré } \\
\text { sin dificultad }\end{array}$ & $\begin{array}{l}\text { Lo conseguiré con } \\
\text { dificultad }\end{array}$ & No lo sé \\
\hline Menos de 200 & 20 & 60 & 20 & 40 & 20 & 40 \\
\hline $200-500$ & 40,3 & 58,2 & 1,5 & 49,2 & 38,5 & 12,3 \\
\hline $500-1000$ & 64,4 & 33,3 & 2,3 & 45 & 42,7 & 12,2 \\
\hline $1000-2000$ & 53,6 & 39,3 & 7,1 & 57,1 & 35,7 & 7,1 \\
\hline Más de 2000 & 79,2 & 20,8 & 0 & 50 & 33,3 & 16,7 \\
\hline
\end{tabular}

Fuente: Elaboración propia.

En cuanto la dificultad para conseguir futuros éxitos académicos es en aquellas poblaciones de entre 1000 y 2000 habitantes donde más se estima que se alcanzarán resultados académicos exitosos, con un 57,1\%, siguiéndole positivamente el resto de po- blaciones: 50\% (más de 2000), 49,2\% (200-500), 45\% (500-1000) y $40 \%$ (menos de 200). Las poblaciones de menos de 200 habitantes son las más dudosas en cuanto a sus éxitos académicos, con un $40 \%$ de alumnado que lo avala.

Tabla 4. Tabla cruzada entre tamaño de la población, sector en el que le gustaría trabajar y sector en donde realmente cree que podrá trabajar.

\begin{tabular}{|c|c|c|c|c|c|c|}
\hline \multirow{3}{*}{ Tamaño de la población } & \multicolumn{6}{|c|}{$\%$ Respuestas } \\
\hline & \multicolumn{3}{|c|}{ Sector en donde le gustaría trabajar } & \multicolumn{3}{|c|}{ Sector en el que realmente creen que trabajará } \\
\hline & Primario & Secundario & Terciario & Primario & Secundario & Terciario \\
\hline Menos de 200 & 35,3 & 20,5 & 44,2 & 21,68 & 20 & 58,32 \\
\hline $200-500$ & 25 & 18,2 & 56,8 & 32,27 & 15,42 & 52,31 \\
\hline $500-1000$ & 28,3 & 21,70 & 50 & 16,63 & 32,24 & 51,10 \\
\hline $1000-2000$ & 18,1 & 41 & 40,9 & 30,55 & 12,35 & 57,10 \\
\hline Más de 2000 & 29,9 & 12,8 & 57,3 & 23,79 & 25,21 & 51 \\
\hline
\end{tabular}

Fuente: Elaboración propia.

En la Tabla 4 se observa que en las poblaciones con más de 2000 habitantes es donde más estudiantes consideran que quieren trabajar en el sector servicios con el 57,3\%, siguiéndole las poblaciones de entre 200 y 500 habitantes con un $56,8 \%$, las poblaciones de entre 500 y 1000 habitantes con un 50\% de los estudiantes y, por último, las poblaciones de menos de 200 habitantes con un $44,2 \%$.

Además, en cuanto a en qué creen que trabajarán en un futuro es también el sector servicios el elegido, siendo las poblaciones de menos de 200 habitantes los más acérrimos a esta creencia con un $58,32 \%$ de estudiantes, seguidos de las poblaciones de entre 1000 y 2000 habitantes con el 57,10\%, las poblaciones de entre 200 y 500 habitantes con el 52,31\% de estudiantes, las poblaciones entre 500 y 1000 con el 51,10\% del alumnado y las poblaciones de más de 2000 habitantes con un $51 \%$ de estudiantes que creen que realmente trabajarán en este sector.

\section{Conclusiones}

El objetivo planteado para esta investigación y cuyos resultados aquí se muestran era conocer las expectativas y creencias que el alumnado de las escuelas rurales presentaba sobre su futuro académico y profesional.

Sin embargo, son muy escasas las investigaciones existentes sobre las expectativas del alumnado rural. Casi todos los trabajos (Abós y López, 2000; Abós, 2007) se centran en el papel del docente rural como protagonista de creencias y expectativas profesionales futuras. Entre algunas de las investigaciones que están relacionadas con la temática de esta investigación encontramos los aportes de Alarcón et al. (2010) con respecto a las aspiraciones del alumnado de educación primaria de escuelas rurales chilenas y de Bachmann y Schiefelbein (2012) y Morales (2006) para las creencias y expectativas de padres y profesorado rurales. Con respecto a estudios centrados en el desarrollo rural y la cultura innovadora con TIC desde la escuela rural podemos mencionar los estudios de Amiguinho (2011), Escribano (2012), Raso y Cáceres (2014) y Del Moral, Villalustre y Neira (2014). Por otro lado, en lo concerniente a la atención de las desigualdades y la educación inclusiva en el medio rural, los proyectos de Vigo (2009) y el estudio de Williamson (2010) son una buena muestra del alcance que la atención de la diversidad puede adquirir en entornos poco favorecidos. Por último, como estudios comparativos centrados 
en el éxito escolar en entornos desfavorecidos o secundarios que superan las expectativas impuestas por el contexto se sitúan los de Luz y Belleï (2004), Casey (2004) y Barley y Beesley (2007).

Tan solo el trabajo de Domingo (2013) se aproximaba a conocer la percepción académica de estos alumnos. Al igual que en el estudio de esta última profesora, los resultados obtenidos en esta investigación recalcan como conclusión prioritaria que la mayoría del alumnado de las escuelas rurales se considera estudiantes exitosos que tendrán un buen futuro académico. Aunque esta afirmación varía en función del número de habitantes del pueblo al que pertenecen. De esta forma, se puede determinar que el número de habitantes del pueblo influye en la creencia que el alumnado presenta de su percepción académica, y en la que se observa que los estudiantes rurales que pertenecen a pueblos con mayor número de habitantes son los que se consideran como mejores estudiantes y como aquellos que presentarán mayores éxitos académicos.

Esta creencia puede deberse a la falta de compañeros para poder medir sus actitudes y competencias y lograr, de este modo, desempeñar estrategias cognitivas de elaboración, organización, inferencia y transferencia fundamentales para el aprendizaje (Enrique y Rentería, 2007), que puedan comparan con las adquiridas por iguales de sus mismas características. La propia complejidad del proceso educativo, en el que se deben asumir responsabilidades de forma independiente, activa y reflexiva (Pereira, 2005), ya hace de aquel un camino a veces duro, que se acentúa si el alumnado no encuentra relaciones afectivas en el que apoyarse y unirse para conjuntamente resolver los problemas educativos que vayan surgiendo.

Por último, se encuentran las mismas dificultades al profundizar en las expectativas y creencias profesionales del alumnado de la escuela rural. Al igual que ocurría con las creencias académicas, en este tema los estudios se han centrado en el maestro de estas escuelas (Bachmann y Schiefelbein, 2012) y no en los alumnos, que son la muestra de esta investigación.

La conclusión principal que se puede destacar en este apartado es que a la mayoría del alumnado de la escuela rural le gustaría optar en un futuro a un trabajo perteneciente al sector servicios, aunque esta conclusión también está influida por el número de habitantes del pueblo en donde estudian, ya que son los estudiantes de pueblos con más habitantes los que en su mayoría presentan esta idea. Para ello también consideran que deben formarse más allá de la enseñanza obligatoria.

El número de habitantes de los pueblos también influye en la creencia de si conseguirán trabajar en este sector o no. Los que están más convencidos de ello son los estudiantes de pueblos de menos de 200 habitantes. Esto puede ser debido al deseo de trabajar en las grandes urbes y dedicarse a actividades no habituales en esos pueblos, en donde en la mayoría de los casos se dedican a la agricultura y a la ganadería. Esta situación proseguiría con las migraciones a las grandes urbes y con el vacío de las áreas rurales (Collantes, 2007), en un proceso continuo que afecta prácticamente a toda el país (Del Molino, 2016), tal vez de manera irreversible.

Entre las futuras líneas de investigación que quedan abiertas se plantea desde la autoría de este artículo profundizar igualmente en las expectativas tanto de profesores como de padres y madres del medio rural para contar con sus relatos de vida y así extraer reflexiones e inferir informaciones relevantes y significativas que puedan ser tratadas analíticamente con posterioridad. Se abre la opción también de diseñar posibles actuaciones que traten de fijar la población al medio rural mitigando sus estereotipos a través de talleres de formación y concienciación con respecto a las posibilidades formativas y laborales del medio rural en el mundo globalizado del siglo XXI. Por último, podría abordarse una reflexión interpretativa sobre el futuro del medio rural y la reorientación de sus patrones culturales y productivos al calor de la terciarización económica y las posibilidades que pueden ofrecer para la mejora de la calidad de vida las nuevas tecnologías, los sectores del turismo rural y del ocio medio-ambiental.

\section{Referencias bibliográficas}

Abós, P. (2007). La escuela rural y sus condiciones ¿tienen implicaciones en la formación del profesorado? Aula Abierta, 35(1,2), 83-90.

Abós, P. y López, A. (2000). Formación inicial del profesorado y Escuela Rural: perspectivas de futuro. Anuario de Pedagogía (Departamento de Ciencias de la Educación, Universidad de Zaragoza), 2, 121-135.

Alarcón, M. ${ }^{a}$ C. et al. (2010). Aspiraciones y expectativas de los alumnos de octavo año básico de la comuna de Villarica: sector urbano-rural. Revista Educativa Sembrando Ideas, 4, 7080.

Alpe, Y. y Fauguet, J.L. (2008). Sociologie de l'école rurale. París: L'Harmattan

Alpe, Y. (2012). Performances scolaires et territoire rural en France. Revue Internationale d'Education de Sèvres, 59, 113-124.

Amiguinho, A. (2011). La escuela en el medio rural: educación y desarrollo local. Profesorado: Revista de curriculum y formación del profesorado, 15(2), 25-37.

Ayuda, I., Pinilla, V. y Sáez, L.A. (2010). Cambio demográfico, despoblación y nuevas políticas: el caso de Aragón en España (CEDDAR, Centro de Estudios sobre Despoblación y Desarrollo en Áreas Rurales), Zaragoza: Facultad de Economía y Empresa, Universidad de Zaragoza.

Bachmann, V., Osses, S. y Schiefelbein, E. (2012). Las Creencias de los profesores rurales: una tarea pendiente para la investigación educativa. Estudios Pedagógicos, 38(1), 297-310.

Barley, Z. y Beesley, A. (2007). Rural School Success: What Can We Learn? Journal of Research in Rural Education, 22(1), 1-16.

Benito, D. (2013). Despoblación, desarraigo y escuela rural: condenados a encontrarse. Encrucijadas, Revista crítica de Ciencias Sociales, 6, 56-69.

Berlanga, S. (2009). La educación rural: entre la realidad y el deseo. Ponencia presentada en las Jornadas sobre Educación en el Medio Rural: encrucijadas y respuestas (mayo, 2009), Teruel, España.

Berlanga, S. (2003). Educación en el medio rural: análisis, perspectivas y propuestas. Zaragoza: Mira.

Bernal, J.L. (2009). Luces y sombras en la escuela rural. Ponencia presentada en las Jornadas sobre Educación en el Medio Rural: encrucijadas y respuestas (mayo, 2009), Teruel, España.

Bernal, J.L. (2006). Comprender nuestros centros educativos. Perspectiva micropolítica. Zaragoza: Mira.

Bernat, A. et al. (2004). Educación y modernización en Aragón en el siglo XX. Zaragoza: Consejo Escolar de Aragón y Facultad de Educación de la Universidad de Zaragoza.

Bernal, A. (2009). Escuela rural e ideologías. Ponencia presentada en las Jornadas sobre Educación en el Medio Rural: encrucijadas y respuestas (mayo, 2009), Teruel, España.

Boix, R. (2011). ¿Qué queda de la escuela rural? Alguna reflexiones sobre la realidad pedagógica del aula multigrado. Profesorado: Revista de curriculum y formación del profesorado, 15(2), 13-23.

Bustos, A. (2011). Escuelas rurales y educación democrática. La oportunidad de la participación comunitaria. REIFOP , 14(2), 105-114. 
Calvo, M. (2003). La educación rural: el horizonte de una nueva cultura. Aula Libre, por una práctica libertaria en la educación, 79, 26-39.

Casey, C. (2004). No hay excusas. Lecciones de 20 escuelas de escasos recursos y alto rendimiento. Washington: Heritage Foundation.

Coladarci, T. (2007). Improving the yield of rural education research: an editor's Swan Song. Journal of Research in Rural Education, 22(3). 20-29.

Collantes, F. (2007). La desagrarización de la sociedad rural española, 1950-1991. Historia agraria, 42, 251-276.

Champollion, P. (2013). Des inégalités d'éducation et d'orientation d'origine territoriale. Paris: L'Harmattan.

Corbett, M. (2007). Travels in space and place: Identity and rural schooling. Canadian Journal of Education, 30(3), 771-785.

Del Moral, M..$^{a}$ E., Villalustre, L. y Neira, M.ํㅗ (2014). Variables asociadas a la cultura innovadora con TIC en escuela rurales. Profesorado: Revista de curriculum y formación del profesorado, $18(3), 9-25$.

Domingo, V. (2013). Origen y evolución del modelo CRA-Colegio Rural Agrupado-. Situación en Aragón y estudio de caso de la realidad turolense. Valencia: Universidad de Valencia [Tesis doctoral].

Enríquez, M.A. y Rentería, P.E. (2007). Estrategias de aprendizaje para la empleabilidad en el mercado de trabajo de profesionales recién egresados. Universitas Pscychologica, 6(1), 89-103.

Escribano, J. (2012). Servicios educativos y sanitarios elementales en el medio rural: percepción social e influencia sobre la calidad de vida. Estudios geográficos, 73 (272), 35-61.

Feu, J. (2004). La escuela rural en España: apuntes sobre las potencialidades pedagógicas, relacionales y humanas de la misma. Revista Digital eRural. Educación, cultura y desarrollo rural, 2(3), 1-13.

Gómez, C. (2007). Lo rural en los planes educativos el Gobierno de Aragón. Revista de Desarrollo Rural y Cooperativismo Agrario, 10, 83-104.

González, J. (2010). Los aspectos positivos y negativos de la educación en la escuela rural. Revista digital Innovación y experiencias educativas, 30,1-13.

Jean, Y. (2007). Géographies de l'école rurale. Acteurs, reseux, territoires. Paris: Ophrys.

Hopkins, T. M. (2005). If you are poor, it is better to be rural: A study of mathematics achievement in Tennessee. Rural Educator, 27(1), 21-33.
Kalaoja, E. y Pietarinen, J. (2009). Small rural primary schools in Finland: A pedagogically valuable part of the school network. International Journal of Educational Research, 48(2), 109119.

Luz, M. 므 y Belleï, C. (coords.) (2004). Escuela efectivas en sectores de pobreza, ¿quién dijo que no se puede? Santiago de Chile: UNICEF.

Manzano, B. y Tomé, M. (2016). The Inclusive Education in Europe. Universal Journal of Educational Research, 4(2), 383-391.

Mallada, L.M. (2012). La dirección de un Centro Rural Agrupado. Revista del Fórum Europeo de Administración de la Educación: Organización y gestión educativa, 6(20), 8-12.

Molino, S. del (2016). La España vacía. Viaje por un país que nunca fue. Madrid: Turner.

Morales, N. (2006). Padres y profesores, dos importantes agentes sociales en las organizaciones escolares españolas y sus interrelaciones en un contexto global. Convergencia, 13(41), 87-116.

Murillo, J.L. (2007). ¿Existe la escuela rural? Aula Libre, por una práctica libertaria en la educación, 85, 6-8.

Pereira, L. (2005). La autorregulación como proceso complejo en el aprendizaje peninsular. Polis, Revista de la Universidad Bolivariana, 4, 11-19.

Raso, F.; Aznar, I. y Cáceres, M. P. (2014). Integración de las tecnologías de la información y comunicación: estudio evaluativo de la escuela rural andaluza (España). Píxel-Bit. Revista de Medios y Educación, 45, 51-64.

Sanz, M. A. (2009). Educación y territorio. Encrucijadas socioculturales. Ponencia presentada en las Jornadas sobre Educación en el Medio Rural: encrucijadas y respuesta (mayo, 2009), Teruel, España.

Sepúlveda, Ma․ P. y Gallardo, M. (2011). La escuela rural en la sociedad globalizada: nuevos caminos para una realidad silenciada. Profesorado: Revista de curriculum y formación del profesorado, 15(2), 141-153.

Vigo, B. (2009). La atención a la diversidad en los núcleos rurales dispersos de la Comunidad Autónoma de Aragón. CEDAR, Centro de Estudios sobre la Despoblación y Desarrollo de Áreas Rurales, 1-7.

Williamson, G. (2010). Todos en el mismo curso. Escuelas multigrado en la educación rural: reflexiones desde algunas experiencias internacionales. Revista Digital eRural, Educación, cultura y desarrollo rural, 7(13-14), 27-34. 